\title{
A Novel Game based Secure Localization Algorithm for Wireless Sensor Networks
}

\author{
Guangyuan Wang ${ }^{1}$, Yongji Ren ${ }^{2 *}$ and Xiaofeng $\mathrm{Xu}^{3}$ \\ ${ }^{1}$ Department of Military Training, Naval Aeronautical and Astronautical \\ University, Yantai 264001, China \\ ${ }^{2}$ Department of Command, Naval Aeronautical and Astronautical University, \\ Yantai 264001, China \\ ${ }^{3}$ Science and Technology on Communication Information Security Control \\ Laboratory, Jiaxing 314033, China \\ *lenglengqiuyu@sina.com
}

\begin{abstract}
Localization in wireless sensor networks (WSNs) is regarded as a pivotal technology which significantly affects the network performance and location-based applications. Since WSNs are always deployed in complex environments, e.g. hostile targets surveillance and tracking, they are more vulnerable to various kinds of hostile attacks in addition to being prone to failure from hostile environmental conditions. Besides the external malicious attacks, the security of localization also suffers from the internal compromised node and its malicious information which are benign originally. Therefore, in this paper, we propose a novel game based secure localization approach for WSNs. First, we transformed the secure localization problem in hostile environments into a game problem. By an improved secure and controlled flood manner, the ordinary nodes successfully constructed a localization nodes domain without external pretended anchors. Then, the to-be-localized nodes initiate the localization game and the neighbor nodes or multi-hop nodes employ some strategies to carry out the appropriate actions as players. Finally, Nash equilibrium methods have been utilized to deal with the game problem and a distributed strategy for players can produce an optimized solution to the WSNs localization. Simulation results show that our scheme is an effective approach to secure localization in wireless sensor networks.
\end{abstract}

Keywords: Wireless Sensor Networks, Secure Localization, Information Game

\section{Introduction}

Wireless sensor networks (WSNs) have drawn more attention in recent years, which offer significant advantages and benefits over traditional wired networks in a wide variety of areas related to environment surveillance and military applications [1]. It largely benefited by the high applicability and economic rationality in harsh environments, which consists of tiny-size, low-cost, easy deployment, self-management, and multifunctional sensor nodes [2-3].

As we known, localization is the pivotal technology for WSNs which significantly affects the network performance and location-based applications, e.g. intrusion detection, target tracking, various military and civilian applications, etc. In a typical network, the majority of sensor nodes are unknown of their positions or coordinates, namely ordinary nodes. The other small part of nodes usually gets position through Global Position System (GPS) or by manual deployments, namely anchor nodes. The ordinary nodes can determine their coordinates based on the localization reference information, e.g. the positions of anchor nodes and the distance constraint relations between ordinary node and 
reference anchors. Extensive research has been conducted in this interesting area and a comprehensive survey is provided in [4-6] and the references therein.

Localizing efficiency and achieving accuracy of node location is a key issue in WSNs. In most traditional localization studies [7], the coordinates of anchor nodes are generally assumed completely correct and reliable and the ordinary nodes can utilize the anchor information safely. However, in the actual situations, security is another important problem for WSNs localization. Since sensor nodes have been always deployed in different kinds of complex environments, e.g. hostile targets surveillance and tracking, they are more vulnerable to various kinds of hostile attacks in addition to being prone to failure from hostile environmental conditions. Though network security has been investigated widely for WSNs, the secure localization which aiming to prevent malicious anchor nodes as well as reference information should be further explored. Because, under hostile localization attacks, some anchor nodes which are benign originally can be compromised and turn into malicious anchor nodes which damage the localization performance specially. In this case, some new approaches should be proposed to eliminate the adverse influence due to malicious anchor nodes attacks and ensure the security and accuracy in WSNs localization.

Therefore, on the basis of analysis of the challenges and the uncertainty of the localization process, this paper proposed a novel game based secure localization algorithm for WSNs. Game theory is a theory of decision making under conditions of uncertainty and interdependence [8]. We transform the secure localization problem in hostile environments into a game issue, which is composed of three kinds of nodes, i.e. ordinary nodes, anchor nodes, and malicious nodes. In this paper, the localization game has three components: a set of players, a set of possible actions for each player and a set of strategies. The ordinary node initiates the localization game and all players can act selfishly to maximize their gains. Nash equilibrium methods have been utilized to deal with the game problem and determine the positions of ordinary nodes. The main research objective is to prevent malicious reference information and seek efficient localization approach with high accuracy and security.

The remainder of this paper is organized as follows. Related works are given in section 2. In section 3, we describe the novel localization algorithm. Simulation setting and result analysis are given in section 4 . And finally, we drew conclusions in section 5.

\section{Related Works}

In [6], Tashnim et al. presented the state-of-art progress of localization algorithms, classification and applications, as well as the future research directions. The authors distinguished the algorithms based on network, indoor application, dependence on anchor nodes, and nodes mobility. From the perspective of parameter definition, classification of algorithms, comparative analysis, application scenarios, and future research directions, this work outlines the whole picture of localization which is different from traditional survey papers. However, the secure localization algorithms and applications haven't been able to discuss enough so that it is difficult to fulfill the demands of the WSNs application security.

Another survey on WSNs localization has been proposed by Han et al. in [9], which classifying the localization algorithms based on static and mobile nodes as well as on range based and range free. Although this work is an excellent summary of recently proposed localization algorithms, it doesn't include the secure localization challenges as well as solutions nor does it provide future research directions. Mesmoudi et al. have discussed localization algorithms in [10], where the algorithms are primarily classified into range free and range based algorithms, each of which are further classified into full schemes and hybrid schemes. Although this work presents a comprehensive analysis of the algorithms, mobility and security issues are not covered extensively. 
In [11], Zhu et al proposed a secure localization approach relying on an attack detection module, which can find out the compromised anchors nodes. First, it harnesses simple geometric triangular rules and an efficient voting technique to enable the attack detection module, which identifies and filters out malicious location references. Then, it uses a secure localization module to compute and cluster certain reference points, which can provide a secure localization service. The position of the concerned regular node is estimated with the centroid of most valuable reference points identified.

Kumar et al [12] proposed a localization methodology that will address the problem of security aspects, where the attackers try to manipulate the estimated location or try to provide false beacons. Firstly, the authors considered the network environment with random node deployment and mobility as these two conditions are less addressed. Further, the proposed algorithm provides low overhead due to the usage of less control messages in a limited transmission range. In addition, they have also proposed a method to detect the malicious anchor nodes inside the network.

Alfao et al. [13] considered the security issue of sensor nodes' localization due to the malicious attacks, where limited trust anchor nodes deployed in the networks. The authors introduced three algorithms to enable the sensor nodes to determine their positions safely. However, the collusion issues of malicious nodes are not covered extensively. Therefore, it would fail when the malicious anchor nodes are in colluding condition.

In [14], Chen et al. proposed to make each locator build a conflicting-set and then the sensor can use all conflicting sets of its neighboring locators to exclude incorrect distance measurements of its neighboring locators. However, the performance of the new localization approach relies primarily on the network conditions, e.g. sparse and dense, quality of link communication. The limitation of the scheme is that it only works properly when the system has no packet loss.

Avinash [15] presented a secure localization method in WSNs using the connected dominating set (CDS) property of a network graph. In this paper, on three incorrect login attempts, a wipe process will be initiated to protect the information onboard the sensor. The malicious node behavior can be a result of either hardware/software malfunction or due to capture and compromise of the node by an adversary. Once captured, the adversary has access to all secure and confidential information on board the captured node. The adversary can compromise the captured node to function as desired. However, the authors assumed that an adversary can only capture but cannot compromise a beacon node, which is not the case for WSNs in practice.

\section{Game Based Secure Localization Algorithm}

To solve these problems, we propose a novel game based secure localization algorithm for WSNs. As stated above, the game theory is a powerful approach to make decision under conditions of uncertainty and interdependence. In this paper, we transform the secure localization problem in hostile environments into a game problem. The main research objective is to prevent the malicious reference information and seek an optimized solution.

Generally speaking, a game has three components, i.e. a set of players, a set of possible actions for each player and a set of strategies. In this paper, the to-be-localized nodes initiate the localization game and the neighbor nodes or multi-hop nodes employ some strategies to carry out the appropriate actions as players. The player's strategy is actually a complete plan of possible actions to be taken when the game played. All players can act selfishly to maximize their payoffs. In this case, a distributed strategy for players can produce an optimized solution to the game [8]. 


\subsection{Preliminaries}

In this paper, we consider three types of nodes in WSNs, namely anchor nodes' $\triangle$ ',

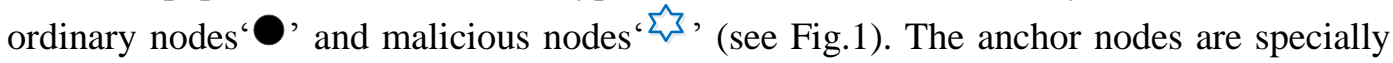
equipped and aware of their coordinates after deployment. The ordinary nodes, whose positions are yet to be discovered, estimate their locations by measuring distances to neighboring or multi-hop anchor nodes. As stated in the beginning, we considered that the wireless sensor network is in a hostile circumstance. That is, there are some malicious attackers during the localization process in the WSNs. The attackers intentionally attack the benign anchor nodes, which are not captured by the attackers successfully. When the attackers compromise a benign anchor node to declare dishonest locations, we call it malicious anchor node. We assume that the physical locations of malicious anchor nodes are not changed, but the malicious anchor nodes can claim fake locations to other nodes.

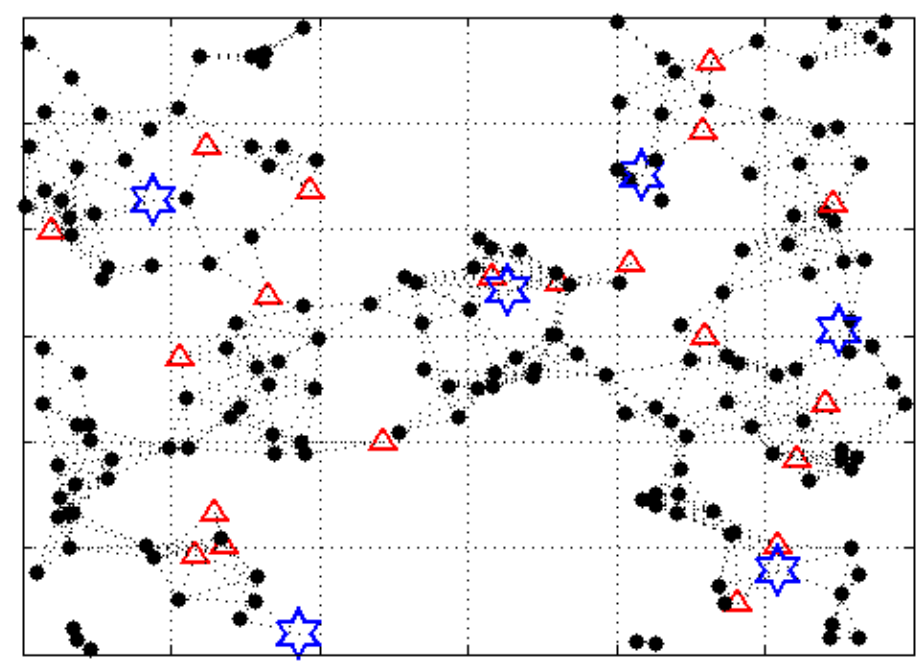

Figure 1. Three Types of Nodes in WSNs

\subsection{Secure and Controlled Localizing Initialization}

In the localization initial phase, the ordinary node broadcasts a localization inviting request to its neighbor nodes. Then, the WSNs can be initialized in an improved secure and controlled flood manner. Since the traditional controlled flood approach has not considered the security issue from external malicious attacks, to eliminate the external pretended anchors in initial phase as much as possible, an appropriative localization hash function has been deployed at each internal benign node to verify the reference node and information received.

A hash function, namely $y=H(x)$, is an algorithm that maps data sets of variable length $x$ to data sets of a fixed length $y$. Considering the limitation of node computing capacity, a basic hash function has been employed in this work [16], which satisfies the following conditions: firstly, give a random $x$, it is easy to obtain $y$ through the function $y=H(x)$; secondly, give a random $y$, it is hard to compute $x$ by the function $y=H(x)$; and thirdly, when $x_{1} \neq x_{2}$, it is impossible to meet the equation $H\left(x_{1}\right)=H\left(x_{2}\right)$. Science each node owns unique identification, the node $I D$ can be defined as $x$ to generate the function $y=H(x)$.

As we known, the localization process which initiated by different ordinary node often overlap. Thus, the neighbor nodes will firstly judge itself whether be overcommitted when received localization inviting request. If the neighbor anchor nodes are free, they respond the agreement message to the ordinary node. Otherwise, they respond the rejection message to the ordinary node. Then, the participating anchor nodes reply the localization reference information, including the node $I D$, measurement distance, $H(I D)$, and 
coordinate of the anchor node, etc. The ordinary node will check whether the hash value $y=H(I D)$ in the localization reference information matches the hash number generated by itself. The values returned by a hash function are called hash values or simply hashes. If the hash value is right, the ordinary node will accept the reference anchor node. Otherwise, the anchor node will be discarded.

Specially, few nodes cannot get enough neighboring anchor nodes due to the sparse feature of network deployment. In this case, it is necessary to make a further messages exchange with multi-hop anchor nodes by corresponding link nodes. Based on the analysis of neighboring nodes information, the ordinary node can figure out whether its neighbor node owns enough anchors. Then the ordinary node can make decision whether or not to send a message to multi-hop nodes. If there are still any nodes which are lack of enough reference nodes, they can select some three-hop anchors which owned better environment by analyzing the two-hop information. At the end, all nodes can get their related information.

By this improved secure and controlled flood manner, the ordinary nodes successfully constructed a localization nodes domain without external pretended anchors, where each node can exchange the reference information from their neighbors or multi-hop reference nodes, e.g. the unique identification of neighbor node, the network density and neighbor list of the anchor node, the coordinates of neighbor anchor, etc.

However, the internal compromised node and its malicious information will continue to disturb the localization security. To solve this problem, in the next phase, we will mix the information game methodology into the localization process.

\subsection{Game Framework}

For a certain node $N_{i}$, we utilize a vector $x_{i}=\left(x_{i, 1}, x_{i, 2}, \cdots, x_{i, n}\right)$ to describe its running states of localization information, e.g. node $I D$, local network density, hash function $H(I D)$, and node coordinate, etc. Each kind of information belongs to one of the following three cases: shared modality, attack modality, and protective modality [17]. The first kind of information, i.e. the shared modality information, has been broadcasted to all the neighbor nodes and exchanged among different kinds of nodes, e.g. the localizing invitation from ordinary node. The second kind of information, i.e. the attack modality information, is detrimental to other benign nodes that declared by the malicious node aiming to disturb the localization process, e.g. the fake locations from malicious anchor nodes. The last kind of information, i.e. the protective modality information, is crucial to the benign nodes that strenuously prevent the interception and manipulation from other malicious nodes, e.g. the location information of benign anchor nodes. Some information which belongs to the protective modality of node $N_{i}$ may be converted into the attack modality information, when the attackers compromised the benign node. Similarly, when the malicious node $M_{j}$ tries to intercept certain information from node $N_{i}$, the node $M_{j}$ should protect its own protective modality information at the mean time during the process of game-play.

After the ordinary node initiated the localization game, the participating anchor nodes will become the game players. Then the localization game domain has been created, involving game sponsor (namely to-be-localized node) and game players (namely participating benign or malicious anchor nodes as well as link nodes). Consider a network consisting of $m$ anchors (involving malicious anchors) and $n$ ordinary nodes which need to be localized. As a result of game-play overlap, there are $n$ localization game domains coexistence. For a certain game domain, we utilize set $V_{k}$ to describe the corresponding nodes.

In the game domain $V_{k}$, each player receiving the localization announcement information calculates its payoff separately. In this game, payoffs represent the motivation of players. A payoff function, describing player's preferences for a given player assigns a 
number for every possible outcome of the game with the property that a higher number implies that the outcome is more preferred [8]. In our localization game framework, the payoff function is given by

$$
P(t, x, u)=\int_{t}^{t_{f}} L(t, x, u) d t+\Psi\left(x_{t_{f}}\right)
$$

with the terminal cost function $\Psi\left(x_{t_{f}}\right)$ and the running cost function

$$
L(t, x, u)=\sum_{j \in V_{k}, j \neq i} c_{i, j}\left(u_{i}\right)-\sum_{j \in V_{k}, j \neq i}\left[a_{i, j} e^{-\theta_{i, j}^{k}\left(x_{i, n}-x_{j, n}, \cdots, x_{i, r_{k}}-x_{j, r_{k}}\right)}-a_{i, j} e^{-\omega_{i, j}^{k}\left(x_{i_{\eta_{k}+1}}-x_{j, r_{k+1}+1} \cdots, x_{i, r_{n}}-x_{j, r_{n}}\right)}\right]
$$

Therein $c_{i, j}\left(u_{i}\right)$ is a control vector of player $N_{i}$, which denotes the control strategies based no negative or positive reaction from the corresponding nodes $N_{j}$ in domain $V_{k}$. If the hash value of node $N_{i}$ matches hash function identification, the control vector $c_{i, j}\left(u_{i}\right)$ will be assigned appropriate positive number. The more positive reactions from other nodes, the larger control vector $c_{i, j}\left(u_{i}\right)$ will be assigned. Otherwise, the value $c_{i, j}\left(u_{i}\right)$ will be negative and grew smaller with increasingly arrival of negative comments. In addition, $a_{i, j} e^{-\theta_{i, j}^{k}}$ is denoted as the attack profit of malicious information when the running states of node $N_{i}$ approaching node $N_{j}$ in localization domain $V_{k}$, and $a_{i, j} e^{-\omega_{i, j}^{k}}$ is denoted as the share modality profit based on convergence of benign reference information from node $N_{i}$ to node $N_{j}$.

The strategy of malicious anchor is to try to make its attack modality information get closer to the corresponding element states of other nodes in localization domain $V_{k}$. This will lead the rise of the attack profit $a_{i, j} e^{-\theta_{i, j}^{k}}$, meanwhile results in the increase of negative reactions to control vector, which causing the final decline of $c_{i, j}\left(u_{i}\right)$ as well as the payoff. In this case, the malicious should share the original reference information rather than the fake positions so as to increasingly enhance the positive comments which can be exchanged in domain $V_{k}$. Otherwise, it will be excluded from the game-play domain.

On the contrary, the strategy of the benign anchor node is to make its share modality information approach to the corresponding element states of other benign nodes in domain $V_{k}$. This will lead the rise of the share modality profit $a_{i, j} e^{-\omega_{i, j}^{k}}$ as well as the payoff function. The more public approval in domain $V_{k}$, the larger control vector and payoffs will be assigned.

As a game player, there are two actions <share, protection> based on their own strategies. In the first round of game-play, while the terminal cost function $\Psi(x)=0$, all players make their action according to the value of their own payoffs, i.e. if $P(t, x, u)>0$, broadcasting a <share> message to all players, or else broadcasting a <protection> message to all players.

For the benign anchor node, the 'share' message means its benign location information. For the malicious nodes, the 'share' messages maybe its attack modality information, i.e. the fake locations of malicious anchor nodes, which depends on the strategy. Whereas the 'protection message means to keep back their own information. Note that whatever the action message submitted to domain $V_{k}$ should include the payoffs $P(t, x, u)$.

Each player in the game domain will increasingly receive the action messages from other game players. Once there is at least one player's payoff that is larger than it has, it should broadcast action messages with < protection > if its previous message is <share >.

According to the Nash equilibrium and calculation demand of game sponsor, the most appropriate players will be employed. At this moment, the game sponsor will broadcasts a candidate list to all players in domain $V_{k}$. Then, as rewards, the value of the terminal cost 
function $\Psi(x)$ of the candidates can be assigned with positive $P(t, x, u)$. Meanwhile, as incentives, the terminal cost function $\Psi(x)$ of the other players fail to be chosen will be assigned with negative $P(t, x, u)$. As the game repeats, the higher the number of participating nodes, the higher will be the utility.

\subsection{Nash Equilibrium and Location Estimation}

The primary strategy of the game sponsor is to determine the more favorable players as localization reference candidates. Different combination of candidates means different localization link from anchor to ordinary node. Thus the further strategy of the game sponsor is to compare the payoffs of different combination of candidates so as to seek most favorable utility for the players, e.g. the load-balanced and stable link for reference information exchange, the elimination of outlier caused by the residuary malicious information, the final secure and accurate localization solution, etc.

Let the admissible control set of localization domain $V_{k}$ be $U^{k}$. A Nash equilibrium is a set of actions of the players such that, any other action chosen by a player does not result in more favorable utility for the players. The admissible control combination $\left(\hat{u}^{1}, \hat{u}^{2}, \cdots, \hat{u}^{k}\right)$ is said to be a Nash equilibrium solution if it satisfies the inequalities

$$
P^{k}\left(0, x, u^{k}\right) \leq P^{k}(0, x,\langle\hat{u} \mid k\rangle)
$$

For the game sponsor, i.e. the to-be-localized node, the Nash equilibrium is that a set of actions result in optimal location estimation while other players choose their favorable control vectors. The location of node $N_{i}$ can be described by $\boldsymbol{X}_{i}=\left[x_{i}, y_{i}, z_{i}\right]^{T}$. The distance from $X_{u}$ to $X_{i}$ can be denoted by $d_{u i}^{I}=\left[d_{u i}^{I-}, d_{u i}^{I+}\right]$, where $d_{u i}^{I-}$ and $d_{u i}^{I+}$ are the corresponding minimal and maximal bounds of $d_{u i}^{I}$, respectively. Each control combination is actually a discriminant relation linking the corresponding anchor node coordinates as well as the ordinary node. The optimum point estimate, i.e. the desired coordinates of ordinary node $X_{u}$, can be obtained by

$$
\begin{gathered}
\hat{U}_{k}=\underset{U_{k}}{\arg \min } \sum_{i=1}^{k}\left(\left\|\hat{X}_{u}-\boldsymbol{X}_{i}\right\|_{2}-d_{u i}\right)^{2} \\
\text { subject to } U_{k} \in V_{k}
\end{gathered}
$$

\section{Performance Evaluation}

In our simulation experiments, 200 nodes with adjustable transmission range $R$ are randomly distributed in a large-scale three dimensional region with a size of $3000 \times 3000 \times 400$, which is composed of three kinds of nodes, i.e. the ordinary nodes ' the anchor nodes ' $\Delta$, , and the malicious nodes ' $s$ ' (see Fig.2). We control the density and connectivity of the network by changing the transmission range $R$ while keeping the area of deployment the same. As seen in Fig.2, the topology of WSNs is not an ideal network situation which suffered from complex environments and randomly deployment in actual application. Part of the anchor nodes which are benign originally have been compromised and turn into malicious anchor nodes which damage the localization performance specially. 


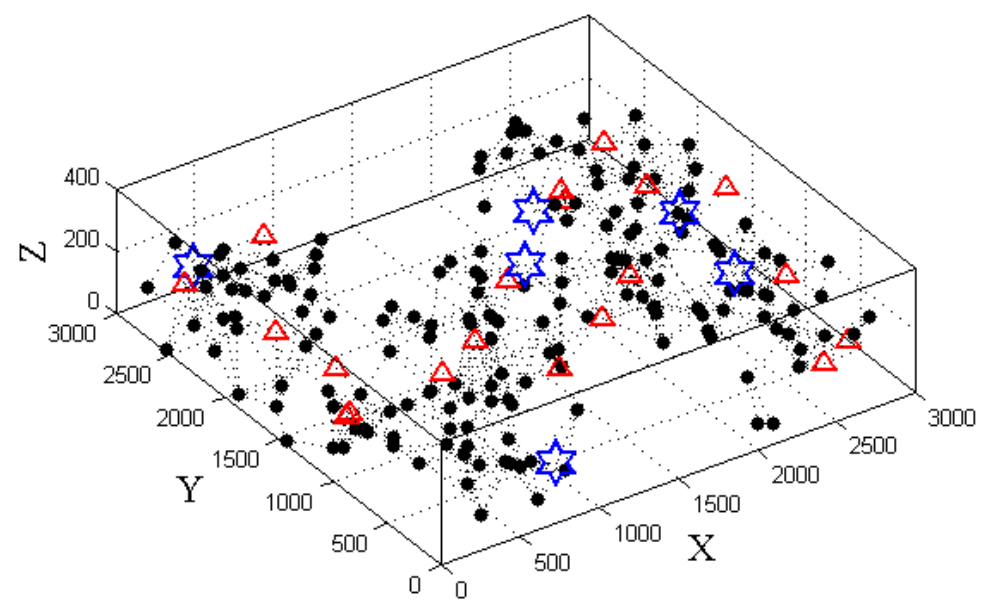

Figure 2. Topology of WSNs

In this paper, we mainly consider three performance metrics: the location coverage rate and the average localization error as well as the percentage of localized nodes with large error, which in the condition of different network connectivity. The first performance metric denotes the percentage of successful localized nodes under the influence of malicious nodes. Average localization error is the average distance between the estimated positions and the real positions of all ordinary nodes. In our simulations, we normalize this absolute localization error to the node transmission range. The third performance metric represents the malicious impact on ordinary nodes. For comparison, we also simulate the DV-distance localization scheme in different conditions.

Fig. 3 plots the performance of location coverage rate and the percentage of localized nodes with large error, with the anchor percentage is $10 \%$ and malicious nodes percentage is $5 \%$. For the first performance metric, the percentage of successfully localized nodes of the DV-distance scheme is higher than our algorithm, when the network connectivity varying from 4 to 14 . The cause of the performance gap is that the malicious nodes participate in DV-distance localization while have been removed from our localization process by game-play. Therefore, though the location coverage rate is higher than us, our percentage of localized nodes with large error is well below it. This indicates the good performance of our proposed scheme in malicious restraint.

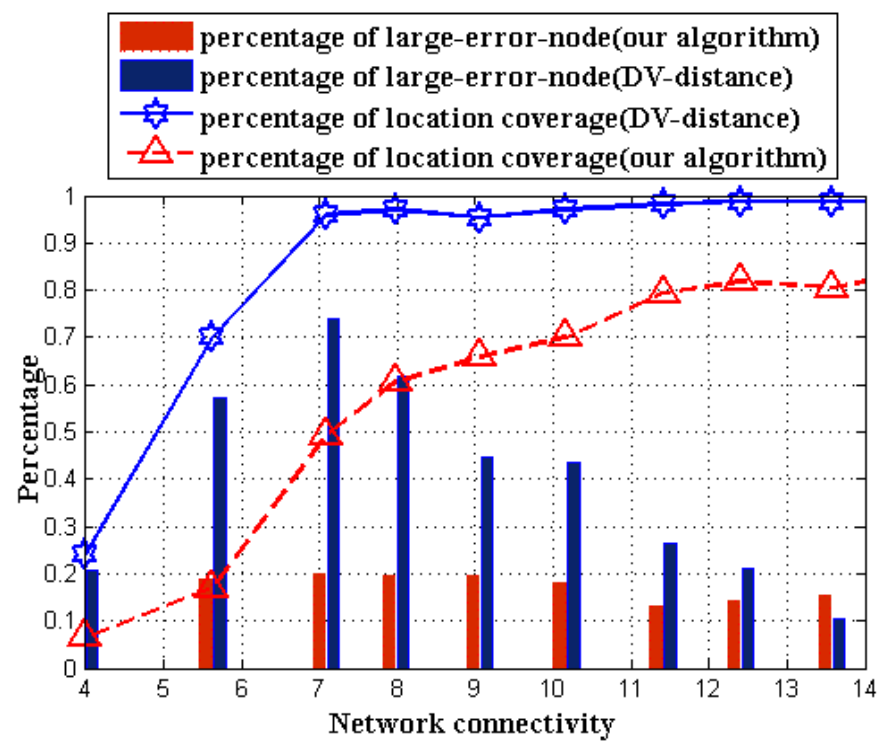

Figure 3. Average Localization Error vs Connectivity 
Fig.4 plots the relationship between the average localization error and network connectivity. We vary the percent of malicious anchor nodes, $5 \%$ and $10 \%$ of total node number, and get the accuracy comparisons with the network connectivity varying from 4 to 14 . We can observe that the average localization error of our scheme obviously outperform DV-distance scheme about 20\% 30\% whether the percent of malicious anchor nodes is $5 \%$ or $10 \%$, while the network connectivity varying from 4 to 10 . It should be noted that our scheme can achieve relatively high and steady output of localization accuracy even with low network connectivity and high malicious node percentage. This indicates the good localization performance of our proposed scheme in harsh environment.

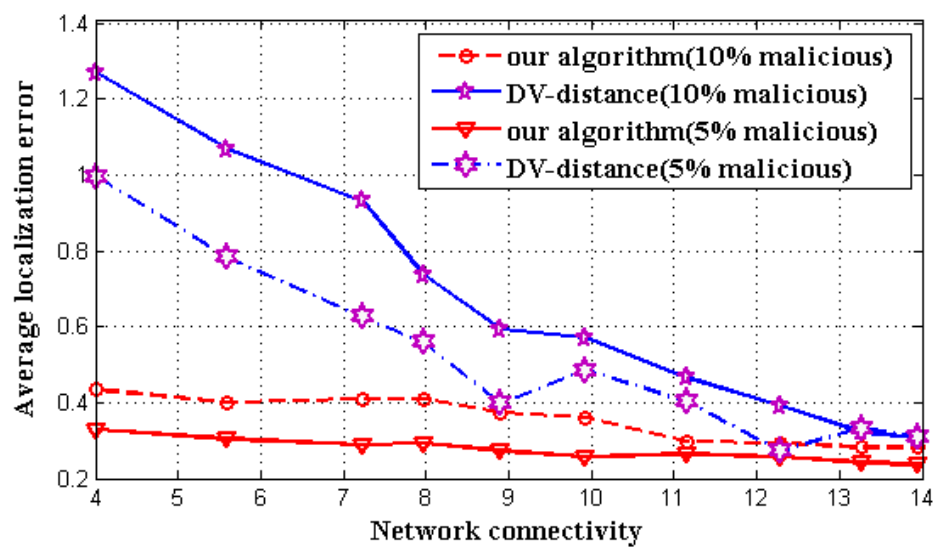

Figure 4. Average Localization Error vs Network Connectivity

\section{Conclusion}

This paper gives a novel attempt to exploit a game methodology to the secure localization as internal compromised and malicious information may happen among sensor nodes. Unlike most of the existing schemes, the to-be-localized nodes initiate the localization game and the neighbor nodes or multi-hop nodes employ some strategies to carry out the appropriate actions as players. Nash equilibrium approach has been utilized to deal with the game problem and a distributed strategy for players can produce an optimized solution to the WSNs localization. Simulation results show that our scheme is an effective approach to secure localization. Our future research work will continue on some more complex factors such as hybrid attack, and we will also consider the load balance and efficiency of WSNs localization.

\section{References}

[1] M.F. Othman, K. Shazali , Wireless sensor network applications: A study in environment monitoring system, Proc. Eng. vol. 41, (2012), pp. 1204-1210 .

[2] J. Yick, B. Mukherjee, D. Ghosal, "Wireless Sensor Network Survey", Computer Networks, vol.52, no.12, (2008), pp.2292-2330.

[3] C. Buratti, A. Conti, D. Dardari, R. Verdone, "An Overview on Wireless Sensor Networks Technology and Evolution", Sensors, vol.9, no.9, (2009), pp. 6869-6896.

[4] G. Mao, Localization Algorithms and Strategies for Wireless Sensor Networks: Monitoring and Surveillance Techniques for Target Tracking: Monitoring and Surveillance Techniques for Target Tracking, IGI Global, (2009).

[5] F. Franceschini, M. Galetto, D. Maisano, L. Mastrogiacomo, "A review of localization algorithms for distributed wireless sensor networks in manufacturing", International Journal of Computer Integrated Manufacturing, (2009), pp. 698-716.

[6] J.S. Tashnim, C.Elkin Chowdhury, V. Devabhaktuni, D. B. Rawat, J. Oluoch, "Advances on localization techniques for wireless sensor networks: A survey, Computer Networks, vol. 110, (2016), pp. 284-305.

[7] A. Pal, "Localization algorithms in wireless sensor networks: Current approaches and future challenges", Netw. Protoc. Algorithms, vol. 2, no. 1, (2010), pp. 45-73.

[8] R. Machado, S. Tekinay, "A survey of game-theoretic approaches in wireless sensor networks", Computer Networks, vol. 52, (2008), pp. 3047-3061. 
[9] G. Han , H. Xu , T.Q. Duong , J. Jiang , T. Hara , Localization algorithms of wireless sensor networks: a survey, Telecommun. Syst., vol.52, no. 4, (2013), pp. 2419-2436 .

[10] A. Mesmoudi, M. Feham, N. Labraoui, Wireless sensor networks localization algorithms: a comprehensive survey, arXiv preprint arXiv:1312.4082 (2013).

[11] W.T. Zhu, Y. Xiang, J.Y. Zhou, R.H. Deng, F. Bao, Secure localization with attack detection in wireless sensor networks, International Journal of Information Security, (2011), pp.155-171.

[12] Kumar. Gulshan, Rai. Mritunjay Kumar, Kim. Hye-Jin, Saha. Rahul, “A Secure Localization Approach Using Mutual Authentication and Insider Node Validation in Wireless Sensor Networks", Mobile Information Systems, (2017).

[13] J.G. Alfao, M. Barbeau, E. Kranakis, "Secure localization of nodes in wireless sensor networks with limited number of truth tellers," in Proceedings of 7th Annual Communication Networks and Services Research Conference, (2009), pp.86-93.

[14] H. Chen, W. Lou, Z. Wang, "Conflicting-set-based wormhole attack resistant localization in wireless sensor networks", In Proc. of the 6th International Conference on Ubiquitous Intelligence and Computing, (2009), pp.296-309.

[15] Avinash Srinivasan, "SecLoc -- secure localization in WSNs using CDS", Security and Communication Networks, vol.4, (2011), pp. 763-770.

[16] N. Yu, L. Zhang, Y. Ren, "A novel D-S based secure localization algorithm for wireless sensor networks", Security Comm. Networks, vol. 7, (2014), pp.1945-1954.

[17] G. Ning, D. Yang, L. Tie, and K.-Y. Cai, "Nash equilibrium of time-delay interaction com-plex networks subject to persistent disturbances", IET Control Theory and Applications, (2012).

\section{Authors}
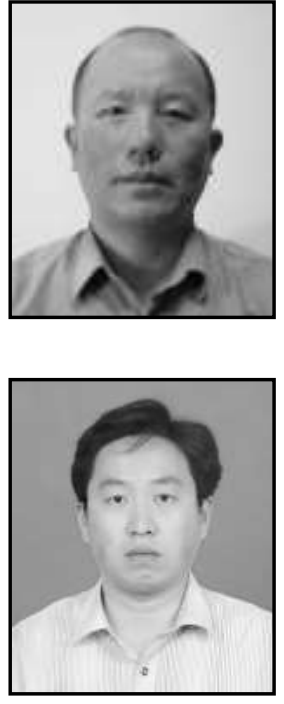

Yongji Ren, He received his Ph.D. degree in School of Instrumentation Science and Opto-electronics Engineering from Beijing University of Aeronautics and Astronautics in 2013. He is a lecturer in the Department of Command at Naval Aeronautical and Astronautical University. His research interests include Wireless Sensor Networks, Underwater Wireless Sensor Networks, Localization and Tracking, Network Security and Information Fusion. 